\title{
The E3 ubiquitin ligase NEDD4 is translationally upregulated and facilitates pancreatic cancer
}

\author{
Min Weng ${ }^{1, *}$, Zhu-Lin Luo ${ }^{2, *}$, Xiao-Ling Wu ${ }^{1}$, Wei-Zheng Zeng ${ }^{1}$ \\ ${ }^{1}$ Department of Gastroenterology, Chengdu Military General Hospital, Chengdu, Sichuan 610083, China \\ ${ }^{2}$ Department of General Surgery, Chengdu Military General Hospital, Chengdu, Sichuan 610083, China \\ * These authors have contributed equally to this work \\ Correspondence to: Wei-Zheng Zeng, email: zhimingzhao616@sina.com \\ Keywords: pancreatic ductal adenocarcinoma, PDAC, NEDD4, PTEN, PI3K/AKT \\ Received: December 08, $2015 \quad$ Accepted: January 06, $2017 \quad$ Published: February 17, 2017
}

\section{ABSTRACT}

Aim: To determine the regulation and function of the neural precursor cell expressed developmentally down regulated protein 4 (NEDD4) in PDAC and to determine its dependency on phosphatase and tensin homolog (PTEN) and PI3K/ AKT signaling.

Methods: We investigated the expression of NEDD4 and the tumor suppressor PTEN in normal immortalized human pancreatic duct epithelial cell line and pancreatic adenocarcinoma (PDAC) cell lines. We further evaluated whether RNAimediated depletion of NEDD4 can attenuate PDAC cell proliferation and migration. We subsequently determined the crosstalk between NEDD4 expression and the PTEN/PI3K/AKT signaling pathway. Finally, we determined the mechanism behind differential NEDD4 protein expression in pancreatic cancer.

Results: The expression of NEDD4 was heterogeneous in PDAC cells, but was significantly higher compared to normal pancreatic ductal epithelial cells. Analogically, PTEN was decreased in the PDAC cells. A combination of MTT assay, wound healing migration assay, and transwell invasion assays confirmed that depletion of NEDD4 decreased the proliferation and migration ability of PDAC cells. Western blot and immunofluorescence results revealed that NEDD4 could affect PTEN/PI3K/AKT signaling pathway in PDAC cells. Polysomal profiling revealed that higher NEDD4 protein expression in PDAC cells was due to undefined mechanism involving translational activation.

Conclusions: Our results reveal a novel mechanism of upregulation of NEDD4 expression in PDAC. Our findings indicate that NEDD4 potentially plays a critical role in activating the PI3K/AKT signaling pathway by negatively regulating PTEN levels in PDAC cells, which promotes pancreatic cancer cell proliferation and metastasis. Therefore, NEDD4 may be a potential therapeutic target in PDAC.

\section{INTRODUCTION}

Pancreatic cancer causes an estimated 227,000 deaths per year worldwide [1-3]. Pancreatic ductal adenocarcinoma (PDAC) is the most common histological type of pancreatic cancer [1] presenting with a highly invasive and metastatic phenotype, which in turn is often responsible for treatment failure and an extremely poor clinical prognosis $[3,4]$. Hence, it is important to identify prognostic and diagnostic markers that can be clinically implemented for optimized treatment strategies for pancreatic cancer patients.

The neural precursor cell-expressed developmentally down regulated gene 4 (NEDD4) was the founding member of the homologous to E6-AP carboxyl terminus (HECT)-type E3 ligases family [5]. Previous studies have shown that ubiquitination does play an important role in controlling the turnover rate, localization and activity 
of cellular proteins [6]. NEDD4 was initially identified as a critical protein in regulating neuronal function and plasticity in the brain [7]. Since NEDD4 itself is regulated by ubiquitination, phosphorylation and other posttranslational modifications, further evidence described the involvement of aberrant NEDD4 expression in cells with damages in the regulatory ubiquitination process. These aberrations lead to malignant transformations, underlying the significance of NEDD4 in tumorigenesis and cancer development $[8,9]$.

PTEN (phosphatase and tensin homolog), a tumor suppressor gene with diverse functions in various tissues, is a negative regulator of the $\mathrm{PI} 3 \mathrm{~K} / \mathrm{AKT}$ signaling pathway. It dephosphorylates PIP3 and thus interferes with AKT activation [10]. PTEN expression is down regulated in several cancer types, including pancreatic cancer [11-13]. Interestingly, recent evidences have suggested NEDD4 as an oncoprotein, since it induces proteasomal degradation of the tumor suppressor PTEN [14-16].

NEDD4 overexpression and its role in cancer growth promotion (independently of PTEN and PI3K/ AKT signaling) were shown in colorectal cancers, in prostate and bladder cancer, and indirectly in esophageal cancer. However, the function and interactions of NEDD4 and PTEN in pancreatic ductal adenocarcinoma (PDAC) still remains elusive. Hence, the objective of the current study was to investigate the regulation and function of NEDD4 in PDAC and to determine its dependency on PTEN and PI3K/AKT signaling.

\section{RESULTS}

\section{NEDD4 and PTEN protein expressions in normal pancreatic ductal epithelium and PDAC cell lines}

We first determined the steady state protein expression of NEDD4 and PTEN in the PDAC cell lines AsPC-1, BxPC-3, Capan-1, PANC-1, and SU.86.86, and a normal immortalized human pancreatic duct epithelial cell line, HPDE6c7. We observed that the expression of both proteins was highly heterogeneous in the PDAC cell lines, with highest NEDD4 expression noted in the PANC-1 cell line (Figure 1A). NEDD4 expression was higher in all the PDAC cell lines compared to the HPDE6c7 cell line.

Interestingly, NEDD4 was expressed at low levels and PTEN was expressed robustly in the normal immortalized human pancreatic duct epithelial cell line, HPDE6c7. Robust PTEN expression was also noted in the PDAC cell lines, BxPC-3 and Capan-1. Cumulatively, this indicated that either there was no correlation between NEDD4 and PTEN expression in the PDAC cell lines, or the correlation is context-dependent and hence not observed universally across all PDAC cell lines.

\section{Depletion of NEDD4 suppresses PTEN/PI3K/ AKT signaling pathway in the PANC-1 cell line and restores an epithelial signature on the cells}

The rational for choosing the PANC-1 cells for subsequent experiments was that it had the highest NEDD4 and lowest PTEN expression and hence provide an ideal scenario to test the functionality of NEDD4 in PDAC. For our subsequent experiment, we transfected NEDD4 siRNA in PANC-1 cells for 48 hours, which showed a significant reduction of NEDD4 protein amount compared to control cells (Figure 1B). It was suggested that NEDD4 is an oncoprotein since it targets PTEN for proteasomal degradation [10, 14-16]. Hence, we investigated the steady state levels of PTEN and PI3K/ AKT signaling pathway members in the parental cells and those harboring the control or NEED4 siRNA. NEDD4 depletion resulted in an increase in the expression of PTEN (Figure 1B). PI3K/AKT signaling pathway expression, which is negatively regulated by PTEN, was altered in our NEDD4 depleted cell lines (Figure 1B). The expression level of total AKT did not change, whereas the expression of phosphorylated AKT was decreased (Figure $1 \mathrm{~B})$ in NEDD4 siRNA-transfectants. This suggests that NEDD4 promotes PI3K/AKT signaling pathway and that PTEN can limit this activation.

We probed for the epithelial cell marker, E-cadherin, and the mesenchymal cell marker, vimentin, in NEDD4 siRNA transfectants. NEDD4 depleted PANC-1 cells resulted in a signature mimicking mesenchymal to epithelial cell transition (MET), with increased E-cadherin and decreased vimentin protein levels (Figure 1B, Figure 2).

\section{Depletion of NEDD4 suppresses the ability of in vitro growth, migration, and invasion in PDAC cells}

It has been reported that NEDD4 might be an important player in malignant transformation processes $[8,9]$. We thus investigated whether depletion of NEDD4 would have an impact on PANC-1 cells ability to grow and to migrate. NEDD4 silencing resulted in decreased proliferation in PANC-1 cells as assessed by the MTT assay (Figure $3 \mathrm{~A}$ ), suggesting that NEDD4 potentiates cell proliferation. Wound-healing experiment results showed that NEDD4 silencing impaired in vitro migration (Figure $3 \mathrm{~B}$ ) and invasion (Figure 3C) in the PANC-1 cells. Given the heterogeneous expression of NEDD4 observed in PDAC cell lines (Figure 1A) and to confirm the observed role of NEDD4 expression in PANC-1 cells, we evaluated the effect of $N E D D 4$ silencing on cell proliferation and in vitro migration and invasion in AsPC-1, SU86.86, BxPC3, and Capan-1 cell lines. RNAi-mediated silencing of NEDD4 resulted in attenuated proliferation (Figure 3A) and in vitro migration (Figure $3 \mathrm{~B}$ ) and invasion (Figure 
3C) in all the PDAC cell lines tested, indicating the prooncogenic role of NEDD4 in PDAC pathology.

\section{Transcriptional upregulation or post-} translational-modification-mediated stabilization does not explain the differential NEDD4 protein expression

We then asked the mechanism behind observed differences of NEDD4 protein expression in the different pancreatic cancer cell lines and the normal nontransformed pancreatic cell line observed in Figure 1A.
Within the cell lines tested, the observed increase in relative NEDD4 protein expression (Figure 1A) was independent of changes in relative expression of steady state expression of NEDD4 messenger RNA (mRNA) (Figure 4A). Furthermore, blocking proteasomal degradation by MG-132 treatment did not result in significant increase in steady-state levels of NEDD4 protein expression in the HPDE6c7 cells or the PANC1 cells (Figure 4B). This suggested that the observed differential NEDD4 protein expression was not due to post-translational stabilization of the NEDD4 protein in the PANC-1 cell line.

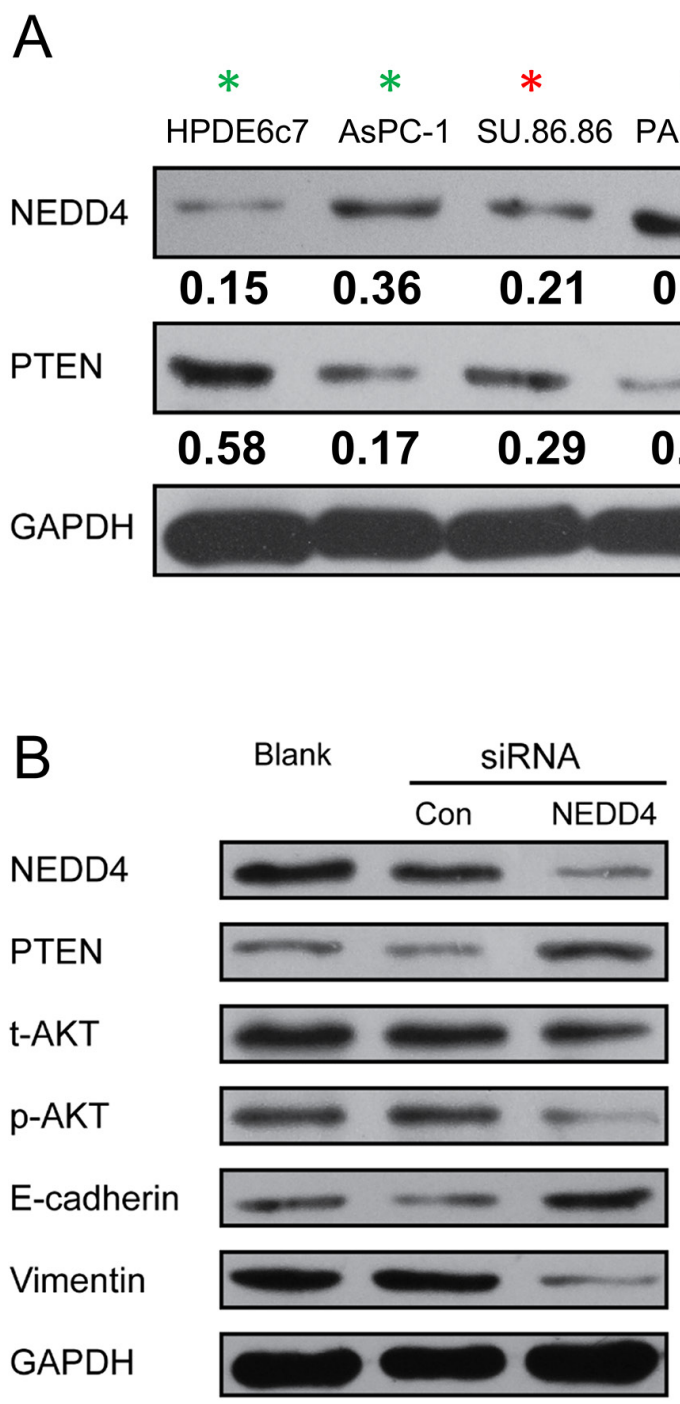

Figure 1: NEDD4 is heterogeneously expressed in PDAC cells and cross-talk with the PTEN/PI3K/AKT signaling pathway. A. The expression of NEDD4 and PTEN in indicated PDAC cell lines, and the normal immortalized human pancreatic duct epithelial cell line, HPDE6c7. The numbers below the NEDD4 and PTEN blots show the relative quantification as obtained by the Image J-mediated densitometry analyses of the blots shown on the left, normalized to GAPDH expression. B. Western blot analysis demonstrating the effect of siRNA-related depletion of NEDD4 on PI3K/Akt activation, PTEN expression, and epithelial (E-cadherin) and mesenchymal cell marker (vimentin) expression. 


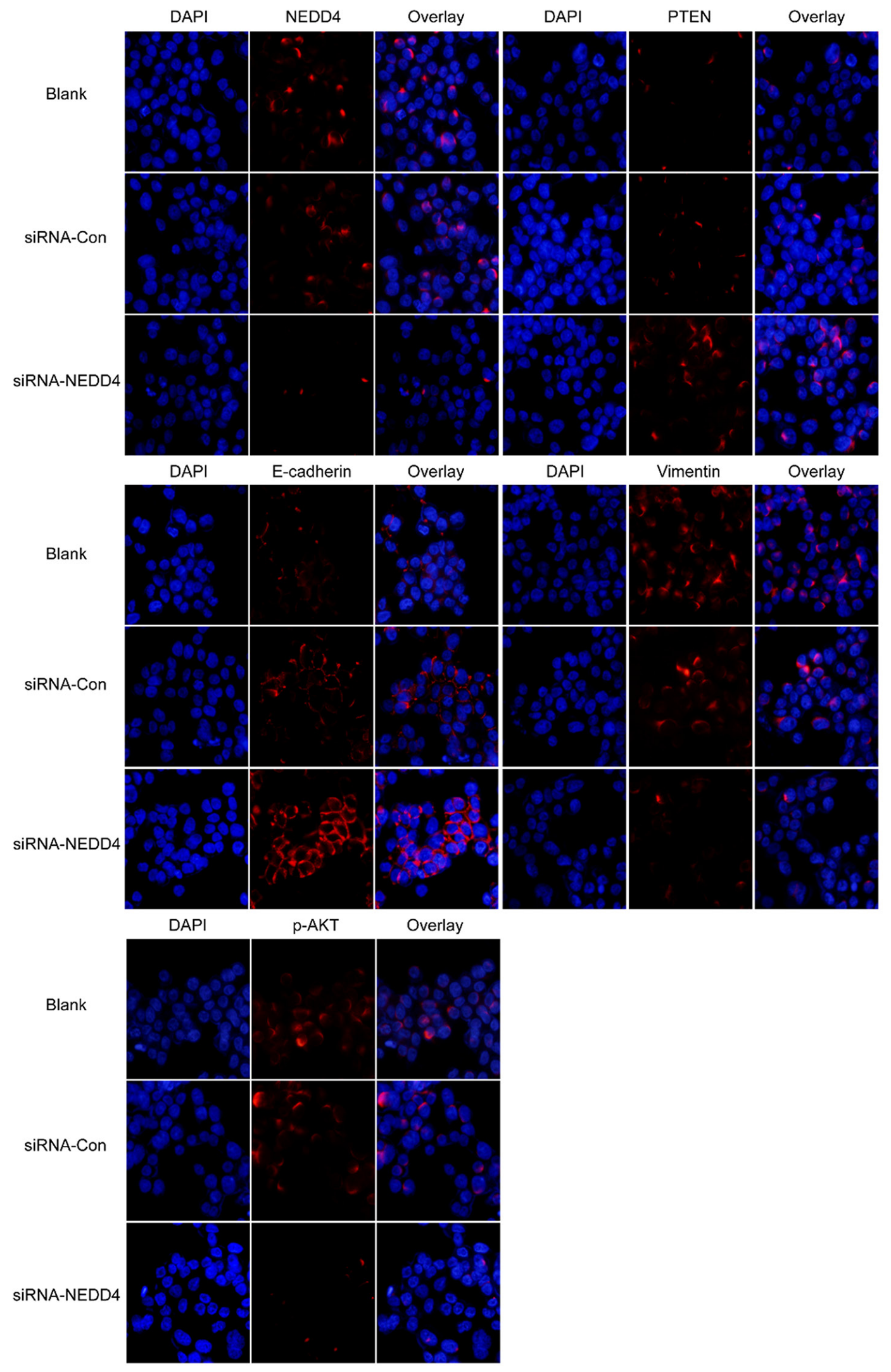

Figure 2: NEDD4 may affect PTEN/PI3K/AKT signaling pathways. NEDD4, PTEN, p-AKT, E-cadherin and vimentin were detected by immunofluorescence. Depletion of NEDD4 negatively affected PTEN expression. A decreased phosphorylation level of AKT associated with acquisition of E-cadherin and loss of vimentin (200x) was also observed. 


\section{NEDD4 is translationally upregulated in PANC- 1 cells}

Since gene expression can be regulated at the level of mRNA transcription, translation, and post-translational modification and we ruled out two of them (Figure 4), we next set out to determine if the observed differences were

A

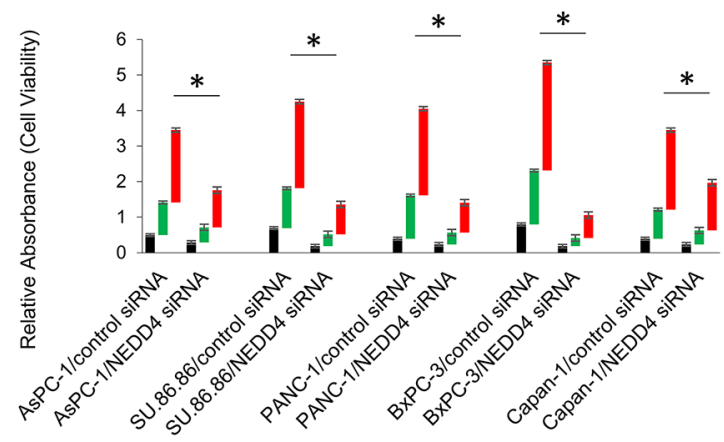

due to differential translational regulation. We determined ribosomal occupancy of NEDD4 mRNA in the HPDE6c7 and the PDAC cell lines. RNA isolated from the different polysome fractions (Figure 5A, 5B) were subjected to quantitative real-time polymerase chain reaction (qRTPCR). There was no difference in global translation efficiency between HPDE6c7 and PANC-1 cells as can

B

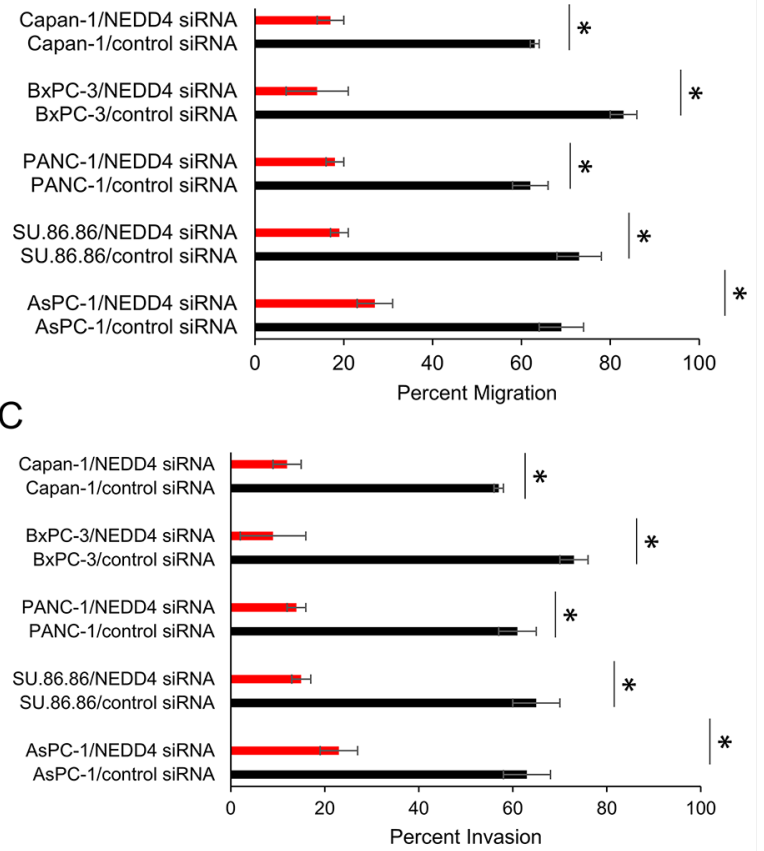

Figure 3: Depletion of NEDD4 inhibited proliferation, migration and invasion in PDAC cells. A. Cell proliferation was analyzed by MTT. Indicated cells were pretreated with NEDD4 siRNA and proliferation was compared at 24, 48, and 72 hours. Depletion led to a significant inhibition of cell proliferation. B, C. Wound-healing and transwell invasion assays, respectively, revealed inhibitory effect on cell migration and invasion ability, similarly to proliferation inhibition experiment. ${ }^{*} P<0.05$.

A

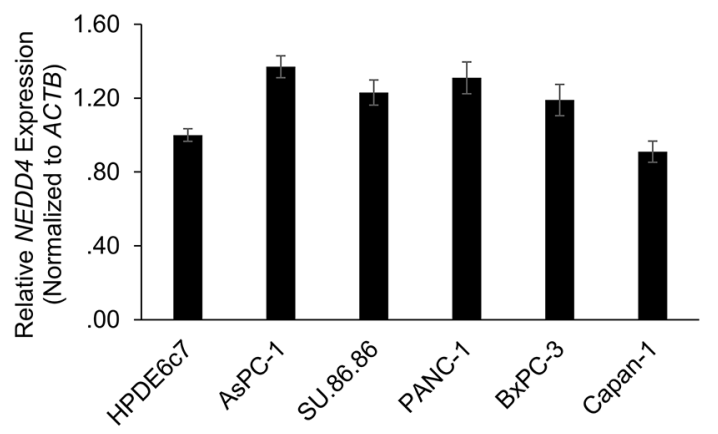

B

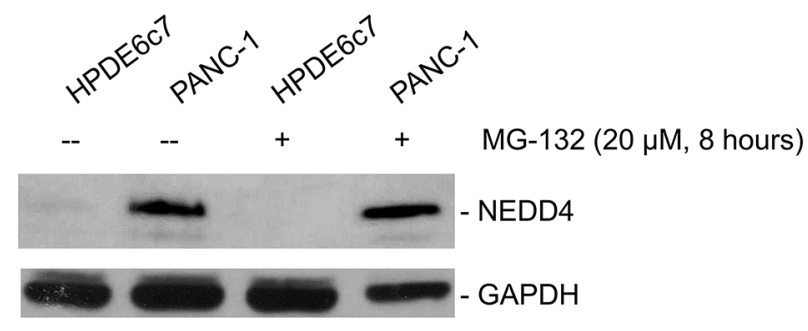

Figure 4: A. Quantitative real-time PCR (qRT-PCR)-based determination of change in steady-state NEDD4 mRNA using total mRNA obtained from indicated cells. All panels are representative of a minimum of three experimental replicates. B. Inhibition of proteasomemediated degradation results did not increase NEDD4 protein levels in the HPDE6c7 cells. Cells were incubated with MG-132 for 8 hours and immunoblotted for the indicated proteins. GAPDH served as a loading control. 
be seen by similar polysomal peaks obtained in the two cell lines. As shown in Figure 5C, NEDD4 mRNA was significantly more polysomally enriched in the PDAC cell lines compared to the HPDE6c7 cells. In addition, the relative polysomal enrichment observed in each of the PDAC cell lines mimicked the relative protein NEDD4 protein expression observed in these cells (Figure 1A). The specificity of the finding was proved by the fact that sequestration into the non-polysomal fractions in HPDE6c 7 was limited to the NEDD4 transcript and the control $A C T B$ had similar pattern of ribosomal occupancy in all the cell lines tested. Taken together, our data shows that the NEDD4 mRNA is translationally activated in pancreatic cancer cells.

\section{DISCUSSION}

Pancreatic cancer is a heterogeneous disease that widely differs in their pathological characteristics and clinical behavior. Almost $60-80 \%$ of patients with pancreatic cancer are metastatic at diagnosis [17]. The mortality is almost identical to the incidence throughout the world, which underlines the high case fatality rate of this aggressive disease. Thus it is imperative to have prognostic and diagnostic markers of human pancreatic cancer that would aid initial detection and also in clinical decision making about treatment modality.

Incidents of PDAC in Asia have been increasing in recent years. Despite intensive research in the diagnostics and therapeutic approaches, such as surgery, chemotherapy, radiotherapy, endocrine and immunotherapy, the clinical outcome and prognosis of PDAC patients remains poor. Expression of NEDD4 was directly associated with cancer progression, particularly metastasis [18-22]. Thus, NEDD4 is proposed as a prognostic biomarker. However, the expression of NEDD4 and its impact on cancer development in PDAC is not known.

NEDD4 is an HECT E3 ubiquitin ligase. It has been shown to participate in the ubiquitination of the tumor suppressor gene PTEN, which in turn suggest that NEDD4 might have a pro-oncogenic role [18-22] NEDD4 was found to be overexpressed in colorectal cancers and promoted tumor cell growth [18]. Inhibition of NEDD4 expression significantly suppressed proliferation of nonsmall cell lung carcinoma (NSCLC) cells and tumor growth in vivo. NEDD4 over-expression augmented the tumorigenicity of lung cancer cells, while the PTEN gene product remained intact [20]. Similarly, it was shown that overexpression of $N E D D 4$ in colon cancer tissues was not
A

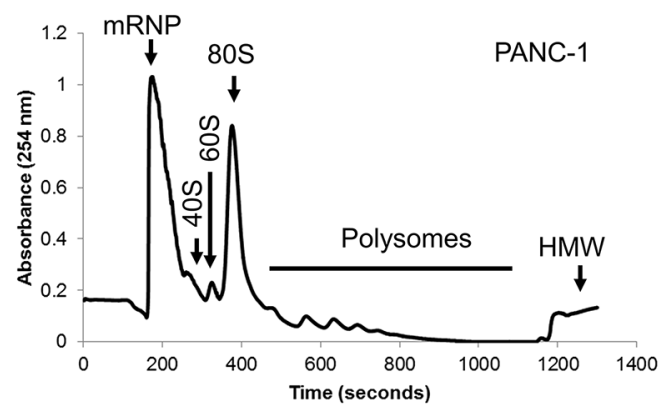

B

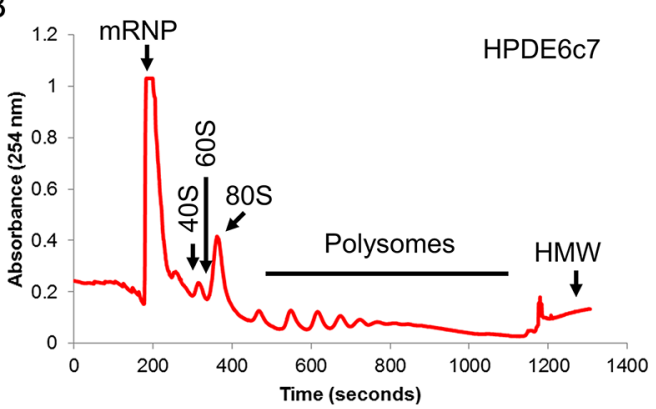

C

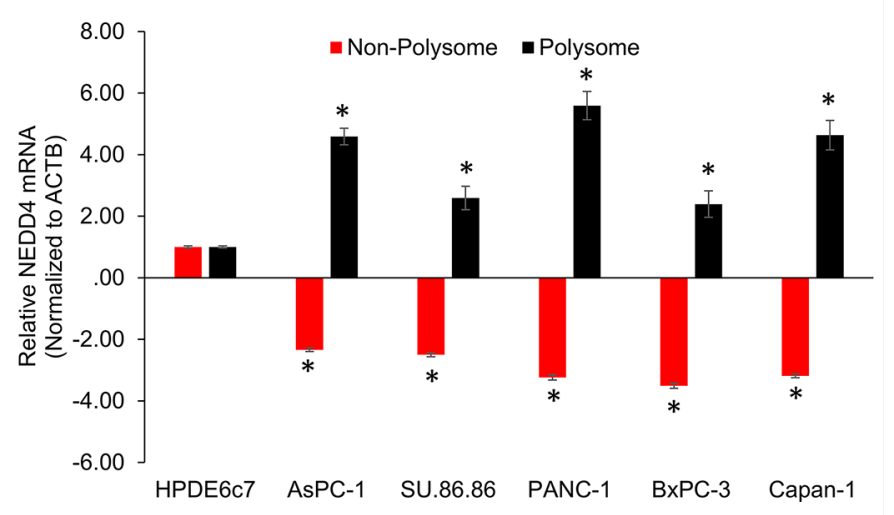

Figure 5: A, B. Representative polysome trace obtained by fractionation of HPDE6c7 (A) and PANC-1 (B) cells. C. QRT-PCR-based determination of change in polyribosomal bound NEDD4 mRNA using non-polyribosomal and polyribosomal enriched mRNA obtained from the indicated cell lines. The data has been plotted relative to relative NEDD4 mRNA in the HPDE6c7 cells. All panels are representative of a minimum of three experimental replicates. ${ }^{*} P<0.05$ compared to HPDE6c 7 cells. 
correlated with down-regulation of PTEN. These results indicate that NEDD4 might be acting through PTENindependent mechanisms. In fact, using conditional knockout mice it was shown that Nedd4-1 is required for axonal growth in murine central nervous system neurons and that PTEN is not a substrate of Nedd4-1 ubiquitination in this context [23]. In fact, PTEN limited Nedd4-1 protein levels by modulating the activity of $\mathrm{mTORC} 1$, a protein complex that controls protein synthesis and cell growth [23]. Our results also suggest that in the context of PDAC, PTEN might not be a substrate of the NEDD4 ubiquitin ligase. Whether the suppression of NEDD4 translation is dictated by PTEN-mediated effect of mTORC1-dependent protein synthesis need to be determined; however, given the heterogeneous expression of PTEN observed in the PDAC cell lines, and observation of high NEDD4 protein even in Capan-1 cells which had high PTEN expression, indicates that there are other potential mechanism for the NEDD4 transcript-specific translational regulation observed in the PDAC cell lines.

In our study, we found that depletion of NEDD4 suppressed the ability of growth and migration in PDAC cell lines. In other words, NEDD4 protein expression could possibly promote the carcinogenesis and cancer progression processes, such as PDAC cell proliferation, migration and invasion. The phosphorylation of AKT was suppressed. This indicates that NEDD4 influences pancreatic ductal adenocarcinoma progression and metastasis through PI3K/Akt signaling pathways. Our data for the first time show that NEDD4 promotes PDAC cell in vitro proliferation, migration, and invasion, which directly relates to tumorigenesis and disease progression. How NEDD4 mediates cell migration and invasion signaling is a crucial and perspective question and requires further investigation.

NEDD4 has been shown to be transcriptionally upregulated by the Forkhead box protein M1B (FoxM1B) in human astrocytes [24, 25]. In pancreatic cancer cells it has been shown that the metastasis suppressor, N-myc downstream regulated gene-1 (NDRG1) inhibits NEDD4 expression [24]. Finally, Casein Kinase I (CKI)mediated hyperphosphorylation of NEDD4 sensitizes the later for degradation by the SCF $\beta$-TRCP ubiquitin E3 ligase complex [26]. Our experiments revealed a novel, translational regulatory mechanism for upregulation of translation of NEDD4 mRNA. To the best of our knowledge, this is the first evidence of post transcriptional regulation of $N E D D 4$ expression. It is imperative that future experiments will have to focus on evaluating the existence of a similar post-transcriptional regulatory mechanism of NEDD4 in patient samples and determining whether the aforementioned mechanisms dictating NEDD4 expression in other contexts are also at interplay. The pleiotropic regulatory mechanisms determining NEDD4 protein expression point to the dynamic importance of inhibiting expression in normal epithelia, and it would not be surprising if multiple mechanisms redundantly regulated expression of NEDD4 in normal pancreas and aberrantly during PDAC.

\section{MATERIALS AND METHODS}

\section{Cell lines, growth conditions, and treatment}

Human PDAC cell lines including AsPC-1, BxPC3, Capan-1, PANC-1, and SU.86.86, and a normal immortalized human pancreatic duct epithelial cell line, HPDEE6c7, were obtained from American Type Culture Collection (ATCC, Manassas, VA, USA). All of the cells except for BxPC-3 and HPDE6c7 were cultured in DMEM containing $4.5 \mathrm{mg} / \mathrm{mL}$ d-glucose and 1-glutamine (Life Technologies, Shanghai, China) supplemented with $10 \%$ FBS. BxPC-3 cells were grown in RPMI 1640 with 10\% fetal bovine serum (FBS) (Lonza, Germany). HPDE6c7 cells were maintained in keratinocyte serum-free medium supplemented by epidermal growth factor and bovine pituitary extract (Life Technologies, Shanghai, China). Cells were kept at $37^{\circ} \mathrm{C}$ under a humidified atmosphere of $5 \%$ carbon dioxide. For the proteasomal inhibition, cells were treated with $20 \mu \mathrm{M}$ of MG-132 (Sigma, Shanghai, China) for the indicated times.

\section{Quantitative real time PCR (qRT-PCR)}

RNA was extracted from indicated cell lines using the Qiagen RNeasy mini kit, and the RT reaction was performed using the ABI TaqMan probes (Assay ID Hs00406454_m1 for NEDD4 and Hs01060665_g1 for $A C T B)$ as per manufacturer recommendations. Data was analyzed by the $-\triangle \Delta C$ t method and normalized to $A C T B$ expression.

\section{Small interfering RNA and transfection}

Indicated cells were transiently-transfected with NEDD4-targeting small interfering RNA (siRNA), sequence with 5'-UUCAAUUGCCAUCUGAAGUUUAUCC-3' (Life Technologies, Shanghai, China). Non-targeting siRNA (Life Technologies, Shanghai, China) was used as control [26]. $5 \times 10^{5}$ cells were seeded in 6 -well plates and grown to $60-80 \%$ confluence. Transient transfection with NEDD4 siRNA into PANC-1 cells using Lipofectamine 2000 (Life Technologies, Shanghai, China) for 72 hours. Simultaneously, non-targeting siRNA was transiently transfected at the same time. In all cases, successful silencing was verified by western blot (Figure 1B, and data not shown).

\section{Cell proliferation assay}

Cell proliferation assay was performed via MTT assay (Sigma-Aldrich, Shanghai, China). $5 \times 10^{3}$ cells 
were plated in 96-well plates and transfected with NEDD4-siRNA or non-targeting siRNA the following day. $20 \mu \mathrm{MTT}(5 \mathrm{mg} / \mathrm{mL})$ were added to each well after 24 , 48 and 72 hours, and incubated for 4 hours. $150 \mu 1$ DMSO were subsequently added to each well and OD values were measured at $570 \mathrm{~nm}$ [27].

\section{Wound healing and invasion assays}

NEDD4-siRNA was transfected into $4 \times 10^{5}$ cells 24 hours post-seeding in 6-well plate. A wound was generated with a pipette tip. Dead cells were removed with PBS. An Olympus BX61 upright microscope was used to take the images of migration ability at 0 hours, 24 hours, 48 hours, and 72 hours. Migration distance was measured by Image J. Cell invasion assay was using transwell chamber assay as per manufacturer recommendations assay (Millipore, Beverly, MA, USA). To rule out compounding effect of NEDD4-siRNA on cell death and its resultant effect on in vitro migration and invasion, the cells in each case were treated with Mitomycin-C before starting each replicate of these experiments.

\section{Western blotting}

Cells were harvested and lysed with RIPA lysis buffer containing protease and phosphatase inhibitor (Thermo Scientific, Beijing, China). Protein concentration was measured using the BCA-kit, and $20 \mu \mathrm{g}$ of each sample was resolved by SDS-PAGE. The primary antibodies included rabbit anti-human NEDD4 (Abcam, ab14592, UK), mouse anti-human AKT, rabbit anti-human p-AKT (Santa Cruz Biotechnology, sc-5298/135650, Dallas, TX, USA), rabbit anti-human PTEN (Abcam, ab32199, UK); mouse anti-human E-cadherin (Abcam, ab1416, UK), mouse anti-human Vimentin (Abcam, ab8979, UK) and mouse anti-human glyceraldehyde 3-phosphate dehydrogenase (GAPDH) (Abcam, ab8245, UK). Optical densitometry analysis was performed using Image $\mathrm{J}$ software. GAPDH served as a loading control in each case.

\section{Polysome analysis}

Polysome analysis was performed as described previously [28, 29]. RNA was extracted from different polysome fractions using Trizol LS reagent (Life Technologies, Shanghai, China) as per manufacturer recommendation. Quantitative real-time PCR was performed as described above.

\section{Immunocytochemistry (ICC)}

Cells were grown on coverslips in 6-well dishes. Cells were transfected with NEDD4-siRNA for 48 hours and washed with PBS. Next, they were fixed with $2 \%$ $(\mathrm{w} / \mathrm{v})$ paraformaldehyde and permeabilized with $1 \%$ (v/v) Triton X-100. Afterwards, they were blocked with $10 \%(\mathrm{w} / \mathrm{v})$ normal goat serum in phosphate-buffered saline (PBS) at room temperature for 1 hour. Finally, they were incubated in one of the primary antibodies overnight at $4^{\circ} \mathrm{C}$. The following day, cells were washed and incubated for 1 hour with Cy3-labeled secondary antibody (Beyotime, Beijing, China) at room temperature, and then co-stained with DAPI (Sigma-Aldrich, Shanghai, China) to visualize the nuclei. Images were obtained using a fluorescence microscope at a magnification of 200x.

\section{Statistical analysis}

Statistical analyses were conducted using SPSS software (version 17.0). All quantitative data were represented as mean \pm standard deviation, determined from at least three independent experiments. Deviations were determined using Student's t test (2-tailed). Statistical evaluation of the data was performed with oneway ANOVA. $P<0.05$ was considered as statistically significant.

\section{CONFLICTS OF INTEREST}

The authors confirm that they do not have any potential conflicts of interest to disclose.

\section{REFERENCES}

1. Vincent A, Herman J, Schulick R, Hruban RH, Goggins M. Pancreatic cancer. Lancet. 2011; 378:607-620.

2. Siegel R, Naishadham D, Jemal A. Cancer statistics, 2013. CA Cancer J Clin. 2013;63: 11-30.

3. Sporn MB. The war on cancer. Lancet. 1996;347:1377-1381.

4. Hanahan D, Weinberg RA. Hallmarks of cancer: the next generation. Cell. 2011;144: 646-674.

5. Donovan P, Poronnik P. Nedd4 and Nedd4-2: ubiquitin ligases at work in the neuron. Int J Biochem Cell Biol. 2013;45:706-710.

6. Kerscher O, Felberbaum R, Hochstrasser M. Modification of proteins by ubiquitin and ubiquitin-like proteins. Annu Rev Cell Dev Biol. 2006;22:159-180.

7. Kumar S, Tomooka Y, Noda M. Identification of a set of genes with developmentally down-regulated expression in the mouse brain. Biochem Biophys Res Commun. 1992;185:1155-1161.

8. Nakayama KI, Nakayama K. Ubiquitin ligases: cell-cycle control and cancer. Nature Rev Cancer. 2006;6:369-381.

9. Chen C, Matesic LE. The Nedd4-like family of E3 ubiquitin ligases and cancer. Cancer Metastasis Rev. 2007;26:587-604.

10. Cantley LC, Neel BG. New insights into tumor suppression: PTEN suppresses tumor formation by restraining the 
phosphoinositide 3-kinase/AKT pathway. PNAS USA. 1999;96:4240-4245.

11. Meng F, Henson R, Wehbe-Janek H, Ghoshal K, Jacob ST, Patel T. MicroRNA-21 regulates expression of the PTEN tumor suppressor gene in human hepatocellular cancer. Gastroenterology. 2007;133:647-658.

12. Wang $\mathrm{H}, \mathrm{Xu} \mathrm{C}$, Kong $\mathrm{X}$, Li X, Kong X, Wang Y, Ding X, Yang Q. Trail Resistance Induces Epithelial-Mesenchymal Transition and Enhances Invasiveness by Suppressing PTEN via miR-221 in Breast Cancer. PLoS One. 2014;9:e99067.

13. Rhee YH, Chung PS, Kim SH, Ahn JC. CXCR4 and PTEN are involved in the anti-metastatic regulation of anethole in DU145 prostate cancer cells. Biochem Biophys Res Commun. 2014; 447:557-562.

14. Wang X, Trotman LC, Koppie T, Alimonti A, Chen Z, Gao Z, Wang J, Erdjument-Bromage H, Tempst P, Cordon-Cardo C, Pandolfi PP, Jiang X. NEDD4-1 is a proto-oncogenic ubiquitin ligase for PTEN. Cell. 2007;128:129-139.

15. Amodio N, Scrima M, Palaia L, Salman AN, Quintiero A, Franco R, Botti G, Pirozzi P, Rocco G, De Rosa N, Viglietto G. Oncogenic role of the E3 ubiquitin ligase NEDD4-1, a PTEN negative regulator, in non-small-cell lung carcinomas. Am J Pathol. 2010:177:2622-2634.

16. Bernassola F, Karin M, Ciechanover A, Melino G. The HECT family of E3 ubiquitin ligases: multiple players in cancer development. Cancer Cell. 2008;14:10-21.

17. Rachagani S, Macha MA, Heimann N, Seshacharyulu P, Haridas D, Chugh S, Batra SK. Clinical implications of miRNAs in the pathogenesis, diagnosis and therapy of pancreatic cancer. Adv Drug Deliv Rev. 2015;81C:16-33.

18. Sakata T, Sakaguchi H, Tsuda L, Higashitani A, Aigaki T, Matsuno K and Hayashi S. Drosophila Nedd4 regulates endocytosis of notch and suppresses its ligand-independent activation. Current Biol. 2014;14:2228-2236.

19. Chen C and Matesic LE. The Nedd4-like family of E3 ubiquitin ligases and cancer. Cancer Metastasis Rev. 2007;26:587-604.

20. Amodio N, Scrima M, Palaia L, Salman AN, Quintiero A, Franco R, Botti G, Pirozzi P, Rocco G, De Rosa N and Viglietto G. Oncogenic role of the E3 ubiquitin ligase NEDD4-1, a PTEN negative regulator, in non-small-cell lung carcinomas. Am J Pathol. 2010;177:2622-2634.

21. Gao C, Pang L, Ren C, Ma T. Decreased expression of Nedd4L correlates with poor prognosis in gastric cancer patient. Medical Oncol. 2012;29:1733-1738.
22. He S, Deng J, Li G, Wang B, Cao Y, Tu Y. Down-regulation of Nedd4L is associated with the aggressive progression and worse prognosis of malignant glioma. Jpn J Clin Oncol. 2012; 42:196-201.

23. Hsia HE, Kumar R, Luca R, Takeda M, Courchet J, Nakashima J, Wu S, Goebbels S, An W, Eickholt BJ, Polleux E, Rotin $\mathrm{D}, \mathrm{Wu} \mathrm{H}$, et al. Ubiquitin E3 ligase Nedd4-1 acts as a downstream target of PI3K/PTENmTORC1 signaling to promote neurite growth. Proc Natl Acad Sci U S A. 2014;111:13205-13210.

24. Dai B, Pieper RO, Li D, Wei P, Liu M, Woo SY, Aldape KD, Sawaya R, Xie K, Huang S. FoxM1B regulates NEDD4-1 expression, leading to cellular transformation and full malignant phenotype in immortalized human astrocytes. Cancer Res. 2010;70:2951-2961.

25. Kovacevic Z, Chikhani S, Lui GY, Sivagurunathan S, Richardson DR. The iron-regulated metastasis suppressor NDRG1 targets NEDD4L, PTEN, and SMAD4 and inhibits the PI3K and Ras signaling pathways. Antioxid Redox Signal. 2013;18:874-887.

26. Liu J, Wan L, Liu P, Inuzuka H, Liu J, Wang Z, Wei W. $\mathrm{SCF}(\beta-\mathrm{TRCP})$-mediated degradation of NEDD4 inhibits tumorigenesis through modulating the PTEN/Akt signaling pathway. Oncotarget. 2014;5:1026-1037. doi: 10.18632/ oncotarget.1675.

27. Eide PW, Cekaite L, Danielsen SA, Eilertsen IA, Kjenseth A, Fykerud TA, Ågesen TH, Bruun J, Rivedal E, Lothe RA, Leithe E. NEDD4 is overexpressed in colorectal cancer and promotes colonic cell growth independently of the PI3K/ PTEN/AKT pathway. Cell Signal. 2013; 25:12-18.

28. Cheng XS, Li YF, Tan J, Sun B, Xiao YC, Fang XB, Zhang XF, Li Q, Dong JH, Li M, Qian HH, Yin ZF, Yang ZB. CCL20 and CXCL8 synergize to promote progression and poor survival outcome in patients with colorectal cancer by collaborative induction of the epithelial-mesenchymal transition. Cancer Lett. 2014;348:77-87.

29. Vyas K, Chaudhuri S, Leaman DW, Komar AA, Musiyenko A, Barik S, Mazumder B. Genome-wide polysome profiling reveals an inflammation-responsive posttranscriptional operon in gamma interferon-activated monocytes. Mol Cell Biol. 2009;29:458-470. 